Igor V. Dolgachev

\title{
On certain families of elliptic curves in projective space $^{\star}$
}

\author{
To the memory of Fabio Bardelli
}

Received: September 18, 2002; in final form: November 10, 2002

Published online: February 16, 2004 - (c) Springer-Verlag 2004

Mathematics Subject Classification (2000). 14E07, 14H25, 14N20

\section{Introduction}

Let $\operatorname{El}\left(n ; p_{1}, \ldots, p_{m}\right)$ be the family of elliptic curves of degree $n+1$ in $\mathbb{P}^{n}$ containing a fixed set of $m$ distinct points $p_{1}, \ldots, p_{m}$. In modern language, $\operatorname{El}\left(n ; p_{1}, \ldots, p_{m}\right)$ is a Zariski-open subset of the fibre of the evaluation map ev : $M_{1, m}\left(\mathbb{P}^{n}, n+1\right) \rightarrow\left(\mathbb{P}^{n}\right)^{m}$, where $M_{1, m}\left(\mathbb{P}^{n}, n+1\right)$ is the space of regular maps of elliptic curves equipped with an ordered set of $m$ points to a curve of degree $n+1$ in $\mathbb{P}^{n}$. Its general fibre, if not empty, is an irreducible variety of dimension $(n+1)^{2}-m(n-1)$. The largest possible $m$ for which the map ev is dominant is equal to $9(n=2,5), 8(n=3,4), n+3(n \geq 6)$. In the last case we show that the general fibre is isomorphic to an open subset of a complete intersection of $n-2$ diagonal quadrics in $\mathbb{P}^{n+2}$. In particular, birationally, it is a Fano variety if $n \leq 6$, a Calabi-Yau if $n=7$, and of general type if $n \geq 8$. The group $g_{n}=(\mathbb{Z} / 2 \mathbb{Z})^{n+2}$ acts naturally in $\mathbb{P}^{n+2}$ by multiplying the projective coordinates with \pm 1 . The corresponding action of a subgroup of index 2 of $g_{n}$ is induced by a certain group of Cremona transformations in $\mathbb{P}^{n}$ which we will describe explicitly.

There are three cases when $\operatorname{El}\left(n ; p_{1}, \ldots, p_{m}\right)$ is of expected dimension 0 . They are $(n, m)=(2,9),(3,8),(5,9)$. It is well known that in the first two cases $\operatorname{El}\left(n ; p_{1}, \ldots, p_{m}\right)$ consists of one point. Less known is the fact that the same is true in the case $(5,9)$. D. Babbage [1] attributes this result to T. G. Room. Apparently it was proven much earlier by A. Coble [2]. We reproduce Coble's proof in the paper. This result implies the existence of a rational elliptic fibration $f: \mathbb{P}^{5}-\rightarrow$ $\operatorname{El}\left(5, p_{1}, \ldots, p_{8}\right)$ which is an analog of the well-known rational elliptic fibrations $\mathbb{P}^{2}-\rightarrow \mathbb{P}^{1}=\operatorname{El}\left(2 ; p_{1}, \ldots, p_{8}\right)$ and $\mathbb{P}^{3}-\rightarrow \mathbb{P}^{2}=\operatorname{El}\left(3 ; p_{1}, \ldots, p_{7}\right)$ defined by the pencil of plane cubics through 8 points and by the net of quadrics through 7

I.V. Dolgachev: Department of Mathematics, University of Michigan, Ann Arbor, MI 48109, USA, e-mail: idolga@umich.edu

* Research partially supported by NSF grant DMS 990780 
points, respectively. We show that its locus of points of indeterminacy is a certain 3-fold, a Weddle variety studied intensively by Coble [4], [5], [6].

I am grateful to D. Eisenbud, S. Mukai and R. Vakil for valuable discussions on the topic of this paper.

\section{Association}

2.1. We start with a reminder of the classical theory of association of finite sets of points. We follow the modern exposition of this theory given in [8].

Let $Z$ be a Gorenstein scheme of dimension 0 over a field $k, \mathcal{L}$ be an invertible sheaf on $Z$ and $V \subset H^{0}(Z, \mathcal{L})$ be a linear system. The duality pairing

$$
H^{0}(Z, \mathcal{L}) \times H^{0}\left(Z, \omega_{Z} \otimes \mathcal{L}^{-1}\right) \rightarrow H^{0}\left(Z, \omega_{Z}\right) \stackrel{\text { trace }}{\longrightarrow} k
$$

allows one to define the subspace $V^{\perp} \subset H^{0}\left(Z, \omega_{Z} \otimes \mathcal{L}^{-1}\right)$. The pair

$$
\left(V^{\perp}, \omega_{Z} \otimes \mathcal{L}^{-1}\right)
$$

is called the Gale transform of $(V, \mathcal{L})$.

2.2. Assume that $Z$ is realized as a closed subscheme of a connected smooth $d$ dimensional scheme $B \subset \mathbb{P}^{n}$. Let $\tau_{Z}$ be its sheaf of ideals in $B$. We have a short exact sequence of sheaves on $B$

$$
0 \rightarrow I_{Z}(1) \rightarrow \mathcal{O}_{B}(1) \rightarrow \mathcal{O}_{Z}(1) \rightarrow 0,
$$

which gives an exact sequence

$$
H^{0}\left(B, \mathcal{O}_{B}(1)\right) \rightarrow H^{0}\left(Z, \mathcal{O}_{Z}(1)\right) \rightarrow H^{1}\left(B, I_{Z}(1)\right) \rightarrow H^{1}\left(B, \mathcal{O}_{B}(1)\right) .
$$

Let us assume that $B$ is embedded by the complete linear system, i.e., the restriction map $H^{0}\left(\mathbb{P}^{n}, \mathcal{O}_{\mathbb{P}}\right) \rightarrow H^{0}\left(B, \mathcal{O}_{B}(1)\right)$ is bijective. Then the image of the map $H^{0}\left(B, \mathcal{O}_{B}(1)\right) \rightarrow H^{0}\left(Z, \mathcal{O}_{Z}(1)\right)$ is equal to $V$. If we also assume that $H^{1}\left(B, \mathcal{O}_{B}(1)\right)=0$, then we will be able to identify $V^{\perp}$ with $H^{1}\left(B, \tau_{Z}(1)\right)^{*}$. Using Serre's duality, we get

$$
V^{\perp} \cong \operatorname{Ext}^{d-1}\left(\mathcal{I}_{Z}(1), \omega_{B}\right)
$$

2.3. In particular, taking $B=\mathbb{P}^{n}$, we can fix a basis in $\operatorname{Ext}^{n-1}\left(\mathcal{I}_{Z}(1), \omega_{\mathbb{P}^{n}}\right)$, so that the linear system $\left|V^{\perp}\right|$ maps $Z$ to a subset $Z^{\text {ass }}$ in the projective space

$$
\mathbb{P}\left(\left(V^{\perp}\right)^{*}\right) \cong \mathbb{P}\left(H^{1}\left(\mathbb{P}^{n}, \tau_{Z}(1)\right)\right) \cong \mathbb{P}^{m-n-2},
$$

where $m=\operatorname{deg} Z:=\operatorname{dim}_{k} H^{0}\left(Z, \mathcal{O}_{Z}\right)$. We will always assume that $m \geq n+3$. The set $Z^{\text {ass }}$ is called the associated set of $Z$. It depends on a choice of a basis in $H^{1}\left(\mathbb{P}^{n}, \tau_{Z}(1)\right)$. If $Z$ is a reduced scheme defined by an ordered set of $m$ distinct points $\left(p_{1}, \ldots, p_{m}\right)$ in $\mathbb{P}^{n}$, then the associated set $Z^{\text {ass }}$ is an ordered set of points $\left(q_{1}, \ldots, q_{m}\right)$ in $\mathbb{P}^{m-n-2}$. 
2.4. Now let $B$ be a curve in $\mathbb{P}^{n}$. Then $Z \subset B$ can be identified with a positive divisor, and

$$
V^{\perp} \cong \operatorname{Ext}^{d-1}\left(\mathcal{I}_{Z}(1), \omega_{B}\right)=H^{0}\left(B, \mathcal{O}_{B}\left(K_{B}+Z-H\right)\right)
$$

where $H$ is a hyperplane section and $K_{B}$ is a canonical divisor. Thus we see that the associated scheme $Z^{\text {ass }}$ is equal to the image of the divisor $Z$ under the map $B \rightarrow \mathbb{P}^{m-n-2}$ given by the complete linear system $\left|K_{B}+Z-H\right|$.

2.5. Remark. Let $Z \subset \mathbb{P}^{n}$ be a reduced scheme consisting of $m$ distinct points $\left(p_{1}, \ldots, p_{m}\right)$. A choice of an order defines a basis in the space $H^{0}\left(Z, \mathcal{O}_{Z}(1)\right)$. Choosing a basis in $H^{0}\left(\mathbb{P}^{n}, \mathcal{O}_{\mathbb{P}^{n}}(1)\right)$ we can represent $Z$ by the matrix $A$ of the linear map $H^{0}\left(Z, \mathcal{O}_{Z}(1)\right)^{*} \rightarrow H^{0}\left(\mathbb{P}^{n}, \mathcal{O}_{\mathbb{P}^{n}}(1)\right)^{*}$ equal to the transpose of the restriction map $H^{0}\left(\mathbb{P}^{n}, \mathcal{O}_{\mathbb{P}}(1)\right)^{*} \rightarrow H^{0}\left(Z, \mathcal{O}_{Z}(1)\right)$. The columns of $A$ are projective coordinates of the points $p_{i}$ 's. Let $B$ be a matrix whose rows form a basis in the nullspace of $A$. Then the columns of $B$ can be chosen as the projective coordinates of the ordered set of points $q_{1}, \ldots, q_{m}$ in $\mathbb{P}^{m-n-2}$. These points represent the associated set of points $Z^{\text {ass }}$ which is well defined up to projective equivalence. This is the original classical definition of association (see [2], [3]). Let $P_{n}^{m}$ be the space of $m$ ordered points in $\mathbb{P}^{n}$ modulo projective equivalence. This is a GIT-quotient with respect to the democratic linearization (i.e the unique linearization compatible with the action of the symmetric group $S_{m}$ ). The association defines an isomorphism of algebraic varieties (see [7]):

$$
\text { ass : } P_{n}^{m} \cong P_{m-n-2}^{m} \text {. }
$$

As far as I know the first cohomological interpretation of the association was given by A. Tyurin [11].

\section{Normal elliptic curves in $\mathbb{P}^{n}$ through $n+3$ points}

3.1. We shall apply 2.4 to the case when $B$ is an elliptic curve embedded in $\mathbb{P}^{n}$ by a complete linear system (a normal elliptic curve of degree $n+1$ ). Let $\operatorname{El}\left(n ; p_{1}, \ldots, p_{m}\right)$ be the set of normal elliptic curves in $\mathbb{P}^{n}$ containing $m \geq n+3$ distinct points $p_{1}, \ldots, p_{m}$. As in the Introduction we consider $\operatorname{El}\left(n ; p_{1}, \ldots, p_{m}\right)$ as an open Zariski subset of the fibre of the evaluation map ev $: M_{1, m}\left(\mathbb{P}^{n}, n+1\right) \rightarrow$ $\left(\mathbb{P}^{n}\right)^{m}$ over $\left(p_{1}, \ldots, p_{m}\right) \in\left(\mathbb{P}^{n}\right)^{m}$. Let $Z$ be the reduced scheme $\left\{p_{1}, \ldots, p_{m}\right\}$ and let $B \in \operatorname{El}\left(n ; p_{1}, \ldots, p_{m}\right)$. We fix a basis in $\mathbb{P}\left(H^{1}\left(\mathbb{P}^{n}, \tau_{Z}(1)\right)\right)$ to identify $Z^{\text {ass }}=\left\{q_{1}, \ldots, q_{m}\right\}$ with an ordered subset of $\mathbb{P}^{m-n-2}$. It follows from 2.4 that $Z^{\text {ass }}$ lies on the image of $B$ under the map $\phi$ given by the complete linear system $|Z-H|$ of degree $m-n-1$. Recall that a choice of a basis in $H^{1}\left(\mathbb{P}^{n}, I_{Z}(1)\right)$ defines a choice of a basis in $H^{0}\left(B, \mathcal{O}_{B}(Z-H)\right)$; so the image of each $B \in \mathrm{El}\left(n ; p_{1}, \ldots, p_{m}\right)$ lies in the same space $\mathbb{P}^{m-n-2}$ and contains $Z^{\text {ass }}$. If $m>n+3$, the map $\phi: B \rightarrow \mathbb{P}^{m-n-2}$ is an embedding. Since the association is the duality, we obtain the following: 
3.2. Theorem. Assume $m>n+3$. Let $Z=\left\{p_{1}, \ldots, p_{m}\right\}$ and $Z^{\text {ass }}=\left\{q_{1}\right.$, $\left.\ldots, q_{m}\right\}$. The association defines an isomorphism of algebraic varieies:

$$
\mathrm{El}\left(n ; p_{1}, \ldots, p_{m}\right) \cong \mathrm{El}\left(m-n-2 ; q_{1}, \ldots, q_{m}\right) .
$$

3.3. Remark. In the case $m=2 n+2$ one can speak about self-associated schemes $Z$, that is, Gorenstein schemes such that $Z^{\text {ass }}=Z$ after an appropriate choice of a basis in $H^{1}\left(\mathbb{P}^{n}, \mathcal{I}_{Z}(1)\right)$. In this case $|H|=|Z-H|$, so that $Z \subset B$ is cut out in $B$ by a quadric. The association isomorphism from Theorem 3.2 is of course the identity.

3.4. Let us consider the exceptional case $m=n+3$. In this case the map $\phi: B \rightarrow$ $\mathbb{P}^{1}$ is of degree 2. It maps $p_{1}, \ldots, p_{n+3}$ to the associated set of points $q_{1}, \ldots, q_{n+3}$ in $\mathbb{P}^{1}$. Conversely, fix a set $\mathcal{Q}=\left\{q_{1}, \ldots, q_{n+3}\right\}$ of distinct points in $\mathbb{P}^{1}$ and let $Z_{Q}$ be the corresponding 0 -dimensional scheme on $\mathbb{P}^{1}$. Take a degree 2 map $\phi: B \rightarrow \mathbb{P}^{1}$ and choose $n+3$ points $\mathcal{P}=\left\{p_{1}, \ldots, p_{n+3}\right\} \subset B$ such that $p_{i} \in \phi^{-1}\left(q_{i}\right)$. Let $Z_{\mathcal{P}}$ be the corresponding 0 -dimensional subscheme of $B$. Since

$$
\phi_{*}\left(\phi^{*}\left(\mathcal{O}_{\mathbb{P}^{1}}(-1)\right) \otimes \mathcal{O}_{Z_{\mathcal{P}}}\right)=\mathcal{O}_{Z_{Q}}(-1),
$$

we can identify the corresponding vector spaces of sections. The associated set $Z_{Q}^{\text {ass }}$ lies on the image of $\mathbb{P}^{1}$ under the map $\mathbb{P}^{1} \rightarrow \mathbb{P}^{n}$ given by the linear system $\left|\mathcal{O}_{\mathbb{P}^{1}}\left(Z_{Q}\right) \otimes \mathcal{O}_{\mathbb{P}^{1}}(-1)\right|$. The linear system

$$
\left|\mathcal{O}_{B}\left(p_{1}+\ldots+p_{n+3}\right) \otimes \phi^{*}\left(\mathcal{O}_{\mathbb{P}^{1}}(-1)\right)\right|
$$

embeds $B$ into the same $\mathbb{P}^{n}$ with $Z_{\mathcal{P}}^{\text {ass }}=Z_{\mathcal{Q}}^{\text {ass }}=Z$. Thus $(B, \mathcal{P})$ defines a point in $\operatorname{El}(n ; Z)$. The double cover $\phi: B \rightarrow \mathbb{P}^{1}$ is defined, up to isomorphism, by a choice of the branch divisor $W \subset \mathbb{P}^{1}$, i.e. an unordered set of 4 distinct points in $\mathbb{P}^{1}$; the latter can be identified with a point in $\mathbb{P}^{4} \backslash \Delta$, where $\Delta$ is the discriminant hypersurface. We will continue to identify $\mathbb{P}^{k}$ with the symmetric product $\left(\mathbb{P}^{1}\right)^{(k)}$. Let $\iota: B \rightarrow B$ be the covering involution. It is clear that the $(B, \mathcal{P})$ and $(B, \iota(\mathcal{P}))$ define the same point in $\operatorname{El}(n ; Z)$. Summarizing we obtain the following:

3.5. Theorem. Let $q_{1}, \ldots, q_{n+3}$ be distinct points in $\mathbb{P}^{1}$ and let $p_{1}, \ldots, p_{n+3}$ be the associated set of points in $\mathbb{P}^{n}$. The variety $\operatorname{El}\left(n ; p_{1}, \ldots, p_{n+3}\right)$ is isomorphic to the fibre of the evaluation map ev $: M_{1, n+3}\left(\mathbb{P}^{1}, 2\right) \rightarrow\left(\mathbb{P}^{1}\right)^{n+3}$ over $\left(q_{1}, \ldots, q_{n+3}\right)$. The forgetting map $M_{1, n+3}\left(\mathbb{P}^{1}, 2\right) \rightarrow M_{1}\left(\mathbb{P}^{1}, 2\right) \cong \mathbb{P}^{4} \backslash \Delta$ restricted to the fibre is a Galois cover with the Galois group $g_{n}$ isomorphic to $(\mathbb{Z} / 2 \mathbb{Z})^{n+2}$.

3.6. Fix a set $\mathcal{P}$ of $m$ points $p_{1}, \ldots, p_{m}$ in $\mathbb{P}^{1}$ and consider the subvariety

$$
X=\left\{A \in \mathbb{P}^{k}: A \cap \mathcal{P} \neq \emptyset\right\} .
$$

It is clear that $X=X_{1} \cup \ldots \cup X_{m}$, where $X_{i}=\left\{A \in \mathbb{P}^{k}: p_{i} \in A\right\}$. This is the image in $\left(\mathbb{P}^{1}\right)^{(k)}$ of a coordinate hyperplane in $\left(\mathbb{P}^{1}\right)^{k}$, and hence is a hyperplane in $\mathbb{P}^{k}$ of effective divisors $D$ of degree $k$ such that $D-p_{i}>0$. Let us identify $\mathbb{P}^{k}$ with $\left|\mathcal{O}_{\mathbb{P}^{1}}(k)\right|$ and let $R=v_{k}\left(\mathbb{P}^{1}\right)$ be the Veronese curve in $\left|\mathcal{O}_{\mathbb{P}^{1}}(k)\right|^{*}=\check{\mathbb{P}}^{k}$. Then $X_{i}$ is the hyperplane in $\left|\mathcal{O}_{\mathbb{P}^{1}}(k)\right|$ corresponding to the point $v_{k}\left(p_{i}\right) \in R$. Thus $X$ is the union of hyperplanes corresponding to $m$ points on $R$. 
3.7. The previous discussion shows that the branch divisor of the map $\operatorname{El}\left(n ; p_{1}\right.$, $\left.\ldots, p_{n+3}\right) \rightarrow \mathbb{P}^{4} \backslash \Delta$ consists of the union of open subsets of $n+3$ hyperplanes corresponding to $n+3$ points on a rational normal curve in $\mathbb{P}^{4}$. Since the fundamental group of the complement of $N$ hyperplanes in a general linear position in $\mathbb{P}^{k}$ is isomorphic to $\mathbb{Z}^{N-1}$, the Galois $(\mathbb{Z} / 2 \mathbb{Z})^{n+2}$-cover $X \rightarrow \mathbb{P}^{4}$ branched along the union of $n+3$ hyperplanes $H_{i}$ is defined uniquely up to an isomorphism. Thus $\operatorname{El}\left(n ; p_{1}, \ldots, p_{n+3}\right)$ is an open subset of $X$. A well-known way to construct $X$ is as follows. Let

$$
l_{i}=\sum_{s=0}^{4} a_{i s} t_{s}=0, \quad i=0, \ldots, n+2
$$

be linear equations defining the hyperplanes $H_{i}$. We assume that $l_{i}=t_{i}, i=$ $0, \ldots, 4$. Consider the map

$$
i: \mathbb{P}^{4} \rightarrow \mathbb{P}^{n+2},\left(t_{0}, \ldots, t_{4}\right) \mapsto\left(l_{0}\left(t_{0}, \ldots, t_{4}\right), \ldots, l_{n+2}\left(t_{0}, \ldots, t_{4}\right)\right) .
$$

Let $\left(x_{0}, \ldots, x_{n+2}\right)$ be projective coordinates in $\mathbb{P}^{n+2}$ and let $\left(y_{0}, \ldots, y_{n+2}\right)$ be projective coordinates in another copy of $\mathbb{P}^{n+2}$. Consider the map $\psi: \mathbb{P}^{n+2} \rightarrow \mathbb{P}^{n+2}$ defined by the formula $\left(x_{0}, \ldots, x_{n+2}\right)=\left(y_{0}^{2}, \ldots, y_{n+2}^{2}\right)$. Then $X$ is isomorphic to the pre-image of $i\left(\mathbb{P}^{4}\right)$ under the map $\psi$. It is easy to see that $X$ is a complete intersection of the quadrics

$$
y_{i}^{2}-\sum_{s=0}^{4} a_{i s} y_{s}^{2}=0, \quad i=5, \ldots, n+2 .
$$

3.8. Corollary. $\operatorname{El}\left(n ; p_{1}, \ldots, p_{n+3}\right)$ is isomorphic to an open Zariski subset of a smooth complete intersection of $n-2$ quadrics in $\mathbb{P}^{n+2}$. In particular, $X$ is birationally of general type for $n>7$, Calabi-Yau for $n=7$, and Fano for $n \leq 6$.

3.9. Remark. The projection from the last point defines a map

$$
\operatorname{El}\left(n ; p_{1}, \ldots, p_{m}\right) \rightarrow \operatorname{El}\left(n-1 ; p_{1}, \ldots, p_{m-1}\right) .
$$

In the case $m=n+3$, this is a finite map of varieties of dimension 4 . I do not know the degree of this map.

\section{Cremona action}

4.1. Let $P_{n}^{m}$ be the space of $m$ ordered points in $\mathbb{P}^{n}$ modulo projective equivalence (see Remark 2.5). Recall that the Cremona action on $P_{n}^{m}$ is a homomorphism of groups:

$$
\mathrm{cr}_{n, m}: W_{n, m} \rightarrow \operatorname{Bir}\left(P_{n}^{m}\right),
$$

where $W_{n, m}$ is the Coxeter group corresponding to the Coxeter diagram defined by a $T_{2, n+1, m-n-1}$ graph (see [7]). Let $s_{0}, \ldots, s_{m-1}$ be its Coxeter generators with $s_{0}$ corresponding to the vertex of the Coxeter diagram with the arm of length 2. 


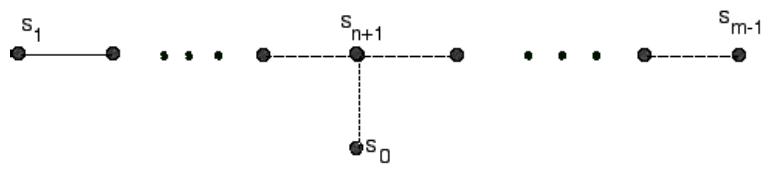

In the action (4.1), the element $s_{0}$ acts by means of the standard Cremona transformation

$$
T: \mathbb{P}^{n}-\rightarrow \mathbb{P}^{n}, \quad\left(z_{0}, \ldots, z_{n}\right) \mapsto\left(z_{1} \cdots z_{n}, z_{0} z_{2} \cdots z_{n}, \ldots, z_{0} z_{1} \cdots z_{n-1}\right),
$$

as follows. Choose an open subset $U$ of $P_{n}^{m}$ representing stable orbits of sets of distinct points $\mathcal{P}=\left(p_{1}, \ldots, p_{m}\right)$ such that $p_{1}, \ldots, p_{n+1}$ span $\mathbb{P}^{n}$. Then one can represent any point $x$ of $U$ with a set $\mathcal{P}=\left(p_{1}, \ldots, p_{m}\right)$ such that

$$
p_{1}=(1,0, \ldots, 0), \ldots, p_{n+1}=(0, \ldots, 0,1) .
$$

Then we define

$$
\mathrm{cr}_{n, m}\left(s_{0}\right)(x)=\left(p_{1}, \ldots, p_{n+1}, T\left(p_{n+2}\right), \ldots, T\left(p_{m}\right)\right) .
$$

The subgroup $S_{m}$ of $W_{n, m}$ generated by $s_{1}, \ldots, s_{m-1}$ acts via a permutation of factors of $\left(\mathbb{P}^{n}\right)^{m}$.

4.2. For any subset $\mathcal{P} \in\left(\mathbb{P}^{n}\right)^{m}$ representing a point $x \in U$ let $\pi_{\mathcal{P}}: X(\mathcal{P}) \rightarrow \mathbb{P}^{n}$ denote the blowing up of $\mathbb{P}^{n}$ with center at $\mathcal{P}$. The Picard group $\operatorname{Pic}(X(\mathcal{P}))$ has a natural basis $e_{\mathcal{P}}=\left(e_{0}, e_{1}, \ldots, e_{m}\right)$, where $e_{0}$ is the class of the pre-image of a hyperplane, and $e_{i}, i>0$, is the class of the exceptional divisor blown-up from the point $p_{i}$. This basis is independent of a choice of a representative of the orbit $x$. The group $W_{n, m}$ acts on $\operatorname{Pic}(X(\mathcal{P}))$ as follows. The subgroup $S_{m}$ acts by permuting $e_{i}, i \neq 0$, and $s_{0}$ acts by

$e_{0} \rightarrow e_{0}-e_{1}-\ldots-e_{n+1}, \quad e_{i} \rightarrow e_{0}-e_{1}-\ldots-e_{n+1}+e_{i}, i=1, \ldots, n+1$,

all $e_{i}$ are invariant for $i>n+1$. We immediately check that all elements $w \in W_{n, m}$ leave the anticanonical class

$$
-K_{X(\mathcal{P})}=(n+1) e_{0}-(n-1) \sum_{i=1}^{n+3} e_{i}
$$

invariant.

If $y=\operatorname{cr}_{n, m}(w)(x)$ and $\mathcal{Q}$ is a representative of $y$, then there is a birational map $f: X(\mathcal{P}) \rightarrow X(\mathcal{Q})$ which is an isomorphism in codimension $\geq 2$ such that

$$
w\left(e_{\mathcal{P}}\right)=f^{*}\left(e_{\mathcal{Q}}\right) .
$$

In particular, if $y=x$, we can choose $\mathcal{Q}=\mathscr{P}$ and obtain a pseudo-automorphism $g$ of $X(\mathcal{P})$ (i.e. a birational automorphism which is an isomorphism in codimension $\geq 2$ ) such that $w=g^{*}$. 
4.3. Recall from Remark 2.5 that the association defines an isomorphism of algebraic varieties

$$
\operatorname{ass}_{n, m}: P_{n}^{m} \rightarrow P_{m-n-2}^{m} \text {. }
$$

It commutes with the Cremona action in the following sense. There is a natural isomorphism of the Coxeter groups $\tau: W_{n, m} \rightarrow W_{n, m-n-2}$ defined by the unique isomorphism of the Coxeter diagrams $T_{2, n+1, m-n-1}$ and $T_{2, m-n-1, n+1}$ which leaves the vertex corresponding to $s_{0}$ fixed. We have

$$
\mathrm{cr}_{n, m}(w)(x)=\mathrm{cr}_{m-n-2, m}(\tau(w))\left(\operatorname{ass}_{n, m}(x)\right) .
$$

4.4. We will be interested in the special case $m=n+3$. In this case $W_{n, m} \cong$ $W\left(D_{n+3}\right)$, the Weyl group of the root system of type $D_{n+3}$. It is known that $W\left(D_{n+3}\right) \cong G_{n} \rtimes S_{n+3}$, where $G_{n} \cong(\mathbb{Z} / 2)^{n+2}$ is generated by the element $w_{1}=s_{0} \circ s_{n+2}$ and their conjugates. Let $x \in P_{n}^{n+3}$ be represented by a point set $\mathcal{P}$ as in (4.2). We also assume that $p_{n+2}=(1, \ldots, 1), p_{n+3}=\left(a_{0}, \ldots, a_{n}\right)$ with $a_{i} \neq 0$. Then

$$
\operatorname{cr}_{n, n+3}\left(w_{1}\right)(x)=\left(p_{1}, \ldots, p_{n+1}, p_{n+3}^{\prime}, p_{n+2}\right),
$$

where $p_{n+3}^{\prime}=\left(1 / a_{0}, \ldots, 1 / a_{n}\right)$. Consider the projective transformation

$$
g: \mathbb{P}^{n} \rightarrow \mathbb{P}^{n}, \quad\left(t_{0}, \ldots, t_{n}\right) \mapsto\left(a_{0} t_{0}, a_{1} t_{1}, \ldots, a_{n} t_{n}\right) .
$$

Then $w(\mathcal{P})=g(\mathcal{P})$, and hence $\mathrm{cr}_{n, n+3}(x)=x$. This shows that $w_{1}$ acts trivially on $P_{n}^{n+3}$. In particular, the Cremona action $\mathrm{cr}_{n, m}$ has the kernel which is a normal subgroup of $W_{n, n+3}$ containing $w_{1}$. It is easy to see that the kernel coincides with $G_{n}$. For example, this follows from (4.3) since $P_{n}^{n+3} \cong P_{1}^{n+3}$. Thus we obtain that $G_{n}$ acts by pseudo-automorphisms of the blow-up $X(\mathcal{P})$.

4.5. Following [4] we show in more details how the group $G_{n}$ acts on $X(\mathcal{P})$. Let $V$ be the vector space over $\mathbb{F}_{2}$ consisting of subsets of $[n+3]=\{1, \ldots, n+3\}$ of even cardinality with the addition defined by $S+S^{\prime}=S \cup S^{\prime} \backslash\left(S \cap S^{\prime}\right)$. Let $A$ be the affine space over $V$ consisting of subsets of odd cardinality. For any $I \in A$ of cardinality $2 k+1$ consider the following divisor class in $X=X(\mathcal{P})$ :

$$
D_{I}=k e_{0}-(k-1) \sum_{i \in I} e_{i}-k \sum_{i \in \bar{I}} e_{i}
$$

where $\bar{I}$ denotes the complementary subset of $[n+3]$. For example, when $k=0$, i.e., $I=\{s\}$ for some $s \in[n+3]$, we get $D_{\{s\}}=e_{s}$.

Lemma 1. There is a unique isomorphism $h: V \rightarrow G_{n}, J \mapsto w_{J}$ such that $w_{\{n+2, n+3\}}=s_{0} \circ s_{n+2}$, and

$$
w_{J}\left(D_{I}\right)=D_{J+I}, \quad \forall J \in V, \forall I \in A .
$$


Proof. First we check this directly for $w_{\{n+2, n+3\}}$ :

$$
D_{I+\{n+2, n+3\}}=s_{0} \circ s_{n+2}\left(D_{I}\right), \quad \forall I \in A .
$$

Then we extend this to all subsets of cardinality 2 of $V$ by defining, for any $\sigma \in S_{n+3}, w_{\sigma(\{n+2, n+3\})}=\sigma \circ w_{n+2, n+3} \circ \sigma^{-1}$ and using that

$$
D_{I+\sigma(\{n+2, n+3\})}=D_{\sigma\left(\sigma^{-1}(I)+\{n+2, n+3\}\right)}=\sigma \circ w_{n+2, n+3} \circ \sigma^{-1}\left(D_{I}\right) .
$$

Finally, we extend the map $h$ to all even subsets by linearity.

4.6. Let $J \in V$ with $\# J=2 k$. Then

$$
w_{J}\left(e_{s}\right)=D_{J \cup\{s\}}=k e_{0}-(k-1) \sum_{i \in J} e_{i}-k \sum_{i \notin J} e_{i}+e_{s},
$$

if $s \notin J$, and

$$
w_{J}\left(e_{s}\right)=D_{J \backslash\{s\}}=(k-1) e_{0}-(k-2) \sum_{i \in J} e_{i}-(k-1) \sum_{i \notin J} e_{i}-e_{s}
$$

otherwise. We have

$$
\begin{gathered}
-K_{X(\mathcal{P})}=(n+1) e_{0}-(n-1) \sum_{i=1}^{n+3} e_{i}=w_{J}\left((n+1) e_{0}-(n-1) \sum_{i=1}^{n+3} e_{i}\right)= \\
(n+1) w_{J}\left(e_{0}\right) \\
-(n-1)\left(k(n+1) e_{0}-((k-1)(n+1)-1) \sum_{i \in J} e_{i}-(k(n+1)-1) \sum_{i \notin J} e_{i}\right) .
\end{gathered}
$$

This gives

$$
w_{J}\left(e_{0}\right)=(k(n-1)+1) e_{0}-(k-1)(n-1) \sum_{i \in J} e_{i}-k(n-1) \sum_{i \notin J} e_{i}
$$

This shows that the pseudo-automorphism of $X_{\mathcal{P}}$ induced by $\mathrm{cr}_{n, m}\left(w_{J}\right)$ is given by the linear system of hypersurfaces of degree $k n-k+1$ passing through the points $p_{i} \notin J$ with multiplicity $(k-1)(n-1)$ and through the points $p_{i} \in J$ with multiplicity $k(n-1)$.

4.7. Assume that $n=2 g-1$ is odd. Applying Lemma 1 we obtain that, for any $I \in A$,

$$
w_{[n+3]}\left(D_{I}\right)=D_{\bar{I}},
$$

and hence

$$
w_{[n+3]}\left(D_{I}\right)+D_{I}=D_{\bar{I}}+D_{I}=g e_{0}-(g-1) \sum_{i=1}^{n+3} e_{i} \in\left|-\frac{1}{2} K_{X(\mathcal{P})}\right| .
$$


The subgroup $G_{n} \subset W_{n, n+3}$ acts on $X_{\mathcal{P}}$ and leaves the half-anticanonical linear system

$$
\left|-\frac{1}{2} K_{X(\mathcal{P})}\right|=\left|g e_{0}-(g-1) \sum_{i=1}^{n+3} e_{i}\right|
$$

invariant. The distinguished element $w_{[n+3]} \in G_{n}$ leaves $2^{g}$ divisors $D_{I}+D_{\bar{I}} \in$ $\left|-\frac{1}{2} K_{X(\mathcal{P})}\right|$ invariant. Coble shows [4] that

$$
\operatorname{dim}\left|-\frac{1}{2} K_{X(\mathcal{P})}\right|=2^{g}-1
$$

and the divisor classes $D_{I}+D_{\bar{I}} \operatorname{span}\left|-\frac{1}{2} K_{X(\mathcal{P})}\right|$. In particular, $w_{[n+3]}$ acts identically in the projective space $\left|-\frac{1}{2} K_{X(\mathcal{P})}\right|$.

4.8. Let $\tilde{W}_{g} \subset X_{\mathcal{P}}$ be the locus of fixed points of $w_{[n+3]}$. This is defined as the closure of the fixed locus of $w_{[n+3]}$ restricted to an invaraint open subset where $w_{[n+3]}$ is defined. The projection $W_{g}$ of $\tilde{W}_{g}$ in $\mathbb{P}^{n}$ is a Weddle variety of dimension $g$. Coble proves that the linear system $\left|-\frac{1}{2} K_{X(\mathcal{P})}\right|$ maps $W_{g}$ to $\mathbb{P}^{2^{g}-1}$ and the image is isomorphic to the Kummer variety of the Jacobian variety $\operatorname{Jac}(C)$ of a hyperelliptic curve $C$ of genus $g$ embedded via $|2 \Theta|$ on $\operatorname{Jac}(C)$. The curve $C$ is the hyperelliptic curve corresponding to $n+3=2 g+2$ points on $\mathbb{P}^{1}$ associated to $\left(p_{1}, \ldots, p_{n+3}\right)$.

4.9. Let $p \in W_{g}$ be a fixed point of $w_{[n+3]}$. Consider the set $\left(p_{1}, \ldots, p_{n+3}, p\right)$ and $w_{[n+3]}$ as an element of $W_{n, n+4}$. Let $\mathcal{Q}=\left(q_{1}, \ldots, q_{n+3}, q\right)$ be the associated set in $\mathbb{P}^{2}$. The projection from $q$ defines $n+3$ points in $\mathbb{P}^{1}$ which are associated to $\left(p_{1}, \ldots, p_{n+3}\right)$ (see [2], [7]). Using (4.3), we see that $\operatorname{cr}_{n, n+4}\left(w_{[n+3]}\right)$ corresponds to $\mathrm{cr}_{2, n+4}\left(w_{0}\right)$ for some $w_{0} \in W_{2, n+4}$. Moreover, we may assume that $\mathrm{cr}_{2, n+4}\left(w_{0}\right)$ defines an automorphism $g$ (in dimension 2 any pseudo-automorphism is an automorphism) of the blow-up $X(Q)$. Since $\mathrm{cr}_{n, n+3}\left(w_{[n+3]}\right)$ fixes the orbit of $\left(p_{1}, \ldots, p_{n+3}\right)$, we see that $\mathrm{cr}_{2, n+4}\left(w_{0}\right)$ leaves the lines $\left\langle q_{i}\right\rangle$ invariant. This implies that the automorphism $g$ is induced by a de Jonquières transformation of the projective plane (see [10, p. 150]). Its fixed set of points is a hyperelliptic curve of degree $g+2$ with $g$-multiple points at $q$ and tangent lines $\left\langle q, q_{i}\right\rangle$. This curve is isomorphic to the curve $C$ from 4.8 . When we vary the point $p$ in the Weddle variety $W_{g}$ the subvariety of $P_{2}^{n+4}$ of associated point sets $\mathcal{Q}$ consists of orbits of points sets $\left(q_{1}, \ldots, q_{n+3}, q\right)$ such that $C$ admits a plane model $C^{\prime}$ of degree $g+2$ with Weierstrass points at $q_{1}, \ldots, q_{n+3}$ and a $g$-multiple point at $q$. The $g$ branches at $q$ define an effective divisor $D$ of degree $g$ on $C$ such that the map $C \rightarrow C^{\prime}$ is given by the linear system $\left|g_{2}^{1}+D\right|$, where $g_{2}^{1}$ is the linear series of degree 2 defined by the hyperelliptic involution. In this way the Weddle variety $W_{g}$ becomes birationally isomorphic to the symmetric product $C^{(g)}$ modulo the hyperelliptic involution, and hence to the Kummer variety of $\operatorname{Jac}(C)$. All of this can be found in $[3, \S 38]$. 
4.10. Let us see how the group $G_{n}$, in its realization as the group of pseudoautomorphisms of the blow-up $X(\mathcal{P})$, acts on the variety $\operatorname{El}\left(n ; p_{1}, \ldots, p_{n}\right)$. For any $B \in \operatorname{El}\left(n ; p_{1}, \ldots, p_{n}\right)$ let $\bar{B}$ be the proper inverse transform under the projection $\pi_{\mathcal{P}}: X(\mathcal{P}) \rightarrow \mathbb{P}^{n}$. Since $\mathcal{O}_{X(\mathcal{P})}\left(e_{0}\right) \cong \pi_{\mathcal{P}}^{*}\left(\mathcal{O}_{\mathbb{P}^{n}}(1)\right)$, we see that $\bar{B}$ is embedded in $\mathbb{P}^{n}$ by the linear system $\left|e_{0}\right|$. It follows from (4.6) that

$$
\begin{gathered}
w_{J}(\bar{B}) \cdot e_{0}=\bar{B} \cdot w_{J}\left(e_{0}\right) \\
=\bar{B} \cdot\left[(k(n-1)+1) e_{0}-(k-1)(n-1) \sum_{i \in J} e_{i}-k(n-1) \sum_{i \notin J} e_{i}\right] \\
=(k(n-1)+1)(n+1)-(k-1)(n-1) 2 k-k(n-1)(n+3-2 k)=n+1 .
\end{gathered}
$$

This shows that $B^{\prime}=\pi_{\mathcal{P}}\left(w_{J}(\bar{B})\right)$ is embedded in $\mathbb{P}^{n}$ by a complete linear system. Similarly we check that

$$
w_{J}(\bar{B}) \cdot e_{i}=\bar{B} \cdot w_{J}\left(e_{i}\right)=1
$$

and hence $B^{\prime}$ passes through the points $p_{1}, \ldots, p_{n+3}$. Thus each $w_{j}$ acts on the variety $\operatorname{El}\left(n, p_{1}, \ldots, p_{n+3}\right)$. We would like to verify that this action corresponds to the action of the Galois group $g_{n}$ of the cover $\operatorname{El}\left(n ; p_{1}, \ldots, p_{n+3}\right) \rightarrow \mathbb{P}^{4}$ from Theorem 3.5. For this we consider the double covers $\phi: B \rightarrow \mathbb{P}^{1}$ and $\phi^{\prime}: B^{\prime} \rightarrow \mathbb{P}^{1}$ defined by the linear systems $\left|\mathcal{O}_{B}(-1) \otimes \mathcal{O}_{B}\left(p_{1}+\ldots+p_{n+3}\right)\right|$ and $\left|\mathcal{O}_{B^{\prime}}(-1) \otimes \mathcal{O}_{B^{\prime}}\left(p_{1}+\ldots+p_{n+3}\right)\right|$, respectively. Each maps the set $\mathcal{P}=$ $\left(p_{1}, \ldots, p_{n+3}\right)$ to the same set of points in $\mathbb{P}^{1}$, the associated set of $\mathcal{P}$. The isomorphism $w_{J}: B \rightarrow B^{\prime}$ defines an isomorphism of the covers, and we have $\phi^{\prime}\left(w_{J}\left(p_{i}\right)\right)=\phi^{\prime}\left(p_{i}\right)$ for each $i=1, \ldots, n+3$. Thus $B$ and $B^{\prime}$ belong to the same fibre of the map $\operatorname{El}\left(n, p_{1}, \ldots, p_{n+3}\right) \rightarrow \mathbb{P}^{4}$.

The homomorphism $G_{n} \rightarrow g_{n}$ which we have just constructed is not injective in the case when $n=2 g-1$ is odd. In fact, the linear system $\left|-\frac{1}{2} K_{X(\mathcal{P})}\right|$ cuts out a $g_{2}^{1}$ on each $\bar{B}$. Since $w_{[n+3]}$ acts identically on $\left|-\frac{1}{2} K_{X(\mathcal{P})}\right|$ it induces an automorphism of $\bar{B}$. Thus $B^{\prime}=B$ in this case. It is clear that the fixed points of $w_{[n+3]}$ on $\bar{B}$ are the points of intersection of $\bar{B}$ and the Weddle variety $W_{g}$. In particular, we obtain

$$
\# \bar{B} \cap W_{g}=4
$$

unless all curves $b$ are contained in $W_{g}$. However, by using the next lemma and induction on $g$ we see that the latter does not happen.

4.11. Lemma. For any $J \in V$ with $\# J=2 k$, let $F_{J}$ be the set of fixed points of the involution $w_{J}$ in $X_{\mathcal{P}}$. Then $\operatorname{dim} F_{J}=k-1$. If $k>2$, the projection map $\pi_{J}$ from the linear space spanned by the points $p_{i}, i \in \bar{J}$ defines a finite map of degree $2^{n+2-2 k}$ from $\pi_{\mathcal{P}}\left(F_{J}\right)$ to the Weddle variety $W_{k-1}$ in $\mathbb{P}^{2 k-3}$ corresponding to the set $\pi_{J}\left(\left\{p_{i}\right\}_{i \in J}\right)$.

Proof. See [4, p. 456]. 
4.12. Theorem. The kernel of the homomorphism $G_{n} \rightarrow g_{n}$ is of order $\leq 2$. It is generated by $w_{[n+3]}$ if $n$ is odd.

Proof. Let $K$ be the kernel of the homomorphism $G_{n} \rightarrow g_{n}$ and $B \in \operatorname{El}\left(n ; p_{1}\right.$, $\left.\ldots, p_{n+3}\right)$. Assume $n$ is odd. Since $K$ leaves the linear system $\left|-\frac{1}{2} K_{X(\mathcal{P})}\right|$ invariant, $K$ leaves the $g_{2}^{1}$ on $B$ cut by this linear system invariant. Thus the image of $K$ in $\operatorname{Aut}(B)$ is equal to the image of $w_{[n+3]}$. If $w_{J} \in K$ acts identically on $B$, then $B$ is contained in the fixed locus $F_{J}$ of $w_{J}$. Hence the union of all curves from $\operatorname{El}\left(n ; p_{1}, \ldots, p_{n+3}\right)$ is contained in $F_{J}$. Let $\# J=2 k$. If $k \leq 2$, this cannot happen since $\operatorname{dim} F_{J}$ is too small. So we may assume that $k>3$. Projecting from the subspace spanned by the points $p_{i}, i \in \bar{J}$, we obtain that the Weddle variety $W_{k-1}$ contains all elliptic curves from $\operatorname{El}\left(2 k-3 ; q_{1}, \ldots, q_{2 k}\right)$. But this contradicts (4.8).

Assume $n$ is even. Assume $B$ is preserved under some $w_{J}$. Since $w_{j}$ sends the set of points $p_{1}, \ldots, p_{n+3}$ to a projectively equivalent set of points, we may assume that $w_{J}$ induces a projective transformation of $B$. Since $B$ is embedded in $\mathbb{P}^{n}$ by a linear system of odd degree, a projective automorphism of $B$ of order 2 cannot be a translation by a 2-torsion point (when we fix a group law on $B$ ). It must fix 4 points on $B$. Now we finish as in the previous case.

4.13. Remark. In the case $n$ is even, the homomorphism $G_{n} \rightarrow g_{n}$ must be injective. However I cannot prove it.

\section{A rational elliptic fibration on $\mathbb{P}^{5}$}

5.1. Now we consider the special case $n=5, m=9$. By Theorem 3.2

$$
\mathrm{El}\left(5 ; p_{1}, \ldots, p_{9}\right) \cong \mathrm{El}\left(2 ; q_{1}, \ldots, q_{9}\right),
$$

where $\mathcal{Q}=\left(q_{1}, \ldots, q_{9}\right)$ is the point set associated to $\mathcal{P}=\left(p_{1}, \ldots, p_{9}\right)$. It is well known that there is a unique nonsingular cubic through a general set of 9 points in $\mathbb{P}^{2}$. Thus we obtain the following:

5.2. Theorem (A. Coble). Let $\left\{p_{1}, \ldots, p_{9}\right\}$ be a general set of 9 points in $\mathbb{P}^{5}$. Then there exists a unique elliptic curve of degree 6 containing these points.

5.3. For a special set of 9 points $\mathcal{Q}$ in $\mathbb{P}^{2}$, the dimension of the linear system of cubics through $Q$ varies from 0 to 5 . However, if we assume that $\operatorname{El}\left(2 ; q_{1}, \ldots, q_{9}\right) \neq \emptyset$, then $\operatorname{dim} \operatorname{El}\left(2 ; q_{1}, \ldots, q_{9}\right) \leq 1$.

5.4. Proposition. Assume that $\operatorname{dim} E l\left(2 ; q_{1}, \ldots, q_{9}\right)=1$. Then the linear system defining the associated set of points $\mathcal{P}=\left(p_{1}, \ldots, p_{9}\right)$ in $\mathbb{P}^{5}$ is equal to the restriction of the complete linear system of conics. In particular, $\mathcal{P}$ is equal to the image of $\mathcal{Q}$ under a Veronese map $v_{2}: \mathbb{P}^{2} \rightarrow \mathbb{P}^{5}$.

Proof. The scheme $Z=\left\{q_{1}, \ldots, q_{9}\right\}$ is cut out by two cubics, and so is arithmetically Gorenstein, i.e., its homogeneous coordinate ring is Gorenstein. Now the assertion follows from Corollary 2.6 of [8]. 
5.5. Remark. The assertion is claimed by Coble in [2]. The proof uses a method which has not been justified so far (see Problem 2.3 in [8]).

5.6. Lemma (A. Coble). Let $E$ be an elliptic curve of degree 6 in $\mathbb{P}^{5}$. Then $E$ is contained in exactly 4 Veronese surfaces.

Proof. Take 9 points on $E$ such that the associated set of points in $\mathbb{P}^{2}$ lies on a pencil of nonsingular cubics $C_{t}$. By Proposition 5.4 and Theorem 3.2, $E$ is the image of some $C_{t}$ under a Veronese map. Thus $E$ is contained in some Veronese surface (this can also be verified by counting constants). Let $V$ be a Veronese surface containing $E$. Then the linear system $|D|$ on $E$ cut out by the complete linear system $L$, where $L$ is an effective generator of $\operatorname{Pic}(V)$, satisfies $|2 D|=\left|\mathcal{O}_{E}(1)\right|$. Also, it comes with a fixed isomorphism $\phi: S^{2}\left(H^{0}\left(\mathcal{O}_{E}(D)\right)\right) \rightarrow H^{0}\left(\mathcal{O}_{E}(2 D)\right)$. Suppose two Veronese surfaces $V$ and $V^{\prime}$ contain $E$ and cut out the same linear system $|D|$ on $E$. Then the two embeddings $E \rightarrow V, E \rightarrow V^{\prime}$ differ by a linear endomorphism $T$ of $H^{0}\left(E, \mathcal{O}_{E}(D)\right)$ such that $S^{2}(T)$ is the identity. This obviously implies that $T$ is the identity, and hence $V=V^{\prime}$. On other hand, if $|D| \neq\left|D^{\prime}\right|$, we can choose an isomorphism $S^{2}\left(H^{0}\left(E, \mathcal{O}_{E}(D)\right)\right) \rightarrow S^{2}\left(H^{0}\left(E, \mathcal{O}_{E}\left(D^{\prime}\right)\right)\right)$ such that the compositions of two embeddings $E \rightarrow\left|\mathcal{O}_{E}(D)\right|^{*}$ and $E \rightarrow\left|\mathcal{O}_{E}\left(D^{\prime}\right)\right|^{*}$ with the corresponding Veronese maps $\left|\mathcal{O}_{E}(D)\right|^{*} \rightarrow\left|\mathcal{O}_{E}(2 D)\right|^{*}$ and $\left|\mathcal{O}_{E}\left(D^{\prime}\right)\right|^{*} \rightarrow$ $\left|\mathcal{O}_{E}\left(2 D^{\prime}\right)\right|^{*}$ are the same. This proves the assertion.

5.7. Let $E \in \operatorname{El}\left(5, p_{1}, \ldots, p_{8}\right)$. Let $V$ be a Veronese surface containing $E$. We may assume that $V$ is the image of the Veronese map $v_{2}: \mathbb{P}^{2} \rightarrow \mathbb{P}^{5}$ and $E=v_{2}(C)$ for some nonsingular plane cubic $C$. Let $q_{1}, \ldots, q_{8}$ be the points on $C$ such that $v_{2}\left(q_{i}\right)=p_{i}, i=1, \ldots, 8$. Choose the ninth point $q_{9}$ such that $\mathcal{Q}=\left\{q_{1}, \ldots, q_{9}\right\}$ is the base locus of a pencil of cubics. Then, by Proposition 5.4 the associated set of points $\mathcal{Q}^{\text {ass }}$ is the image under a Veronese map. We can fix the Veronese map by requiring that $v_{2}\left(p_{i}\right)=q_{i}, i=1, \ldots, 8$. Let $v_{2}\left(q_{9}\right)=p_{9}$. Then by Theorem 3.2, $\operatorname{El}\left(5 ; p_{1}, \ldots, p_{9}\right) \cong \operatorname{El}\left(2 ; q_{1}, \ldots, q_{9}\right)$ is an open subset of $\mathbb{P}^{1}$. We want to show that the point $p_{9}$ is on the Weddle variety $W_{3}$ corresponding to the set of points $\left(p_{1}, \ldots, p_{8}\right)$.

5.8. Lemma. Let $\left(q_{1}, \ldots, q_{9}\right)$ be an ordered set of base points of an irreducible pencil of cubic curves. Then there exists a unique plane curve $C$ of degree 5 with a triple point at $q_{9}$ such that the lines $\left\langle q_{i}, q_{9}\right\rangle$ are tangents to $C$ at $q_{i}$. Conversely, given such a curve $C$, there exists a pencil of cubic curves with base points at $q_{1}, \ldots, q_{9}$.

Proof. We give a modern version of the proof given by Coble in $[3, \S 38]$. Let $X$ be the blow-up of the points $q_{1}, \ldots, q_{9}$. This has a structure of an elliptic surface together with the zero section defined by the exceptional curve $E_{9}$ blown up from $q_{9}$. Let $F$ be the divisor class of a fibre and $S$ be the divisor class of the proper transform of a line through the point $q_{9}$. Then $|F+S|$ defines a double cover $f: X \rightarrow \mathbb{P}^{1} \times \mathbb{P}^{1}$ such that the pre-image of the first ruling is equal to the pencil $|F|$, and the pre-image of the second ruling is equal to the pencil $|S|$. The 
branch curve $B$ of $f$ is a curve of bi-degree $(4,2)$. It is a hyperelliptic curve of genus 3. Its pre-image in $X$ is equal to $2 R$, where $R$ is isomorphic to $B$. On each nonsingular fibre $F$ there are two ramification points of the pencil $|S|$ restricted to $F$. The curve $R$ is equal to the locus of these points when $F$ varies in the pencil. Similarly, for each member of $|S|$ there are two ramification points of the restriction of the pencil $|F|$, and these ramification points are also on $R$.

Let us show that the image of $R$ in $\mathbb{P}^{2}$ is the hyperelliptic curve $C$ from the assertion of the lemma. The image $\bar{E}_{9}$ of $E_{9}$ is a curve of bi-degree $(1,1)$ such that $f^{-1}\left(\bar{E}_{9}\right)=E_{9}+E_{9}^{\prime}$, where $E_{9}^{\prime}$ is the image of $E_{9}$ under the involution of $X$ defined by the double cover $f$. Since $\bar{E}_{9}$ is tangent everywhere to the branch curve $R$, we see that $E_{9}$ intersects $R$ at 3 points. Thus $C$ has a triple point at $q_{9}$. Let $E_{i}$ be the exceptional curve blown up from $q_{i}$ and let $S_{i}$ be the proper inverse transform of the line $\left\langle q_{i}, q_{9}\right\rangle$. Then $E_{i}+S_{i} \in|S|$, and $f\left(E_{i}\right)=f\left(S_{i}\right)$ is a fibre of the second ruling of $\mathbb{P}^{1} \times \mathbb{P}^{1}$ which is tangent to $B$ at a point $P$ such that $f^{-1}(P)=E_{i} \cap S_{i}$. This shows that $E_{i} \cap S_{i} \cap R \neq \emptyset$, and hence $C$ is tangent to $\left\langle q_{i}, q_{9}\right\rangle$ at $q_{i}$. Thus $C$ satisfies all the properties from the assertion of the lemma. Let us show its uniqueness. Suppose $C^{\prime}$ is another curve satisfying the same properties. Let $R^{\prime}$ be its proper inverse transform on $X$. We have $R \cdot R^{\prime}=25-9-16=0$, and $R^{2}=R^{\prime 2}=25-9-8=8$. This contradicts the Hodge Index Theorem.

It remains to prove the converse. We will be brief, letting the reader fill in the details. Let $R$ be the proper inverse transform of $C$ in $X$. Then the linear system $|R-S|$ defines a double cover $f: X \rightarrow \mathbb{P}^{1} \times \mathbb{P}^{1} \subset \mathbb{P}^{3}$. If the restriction of $f$ to $S$ is of degree 1 , then its image is a plane section of $\mathbb{P}^{1} \times \mathbb{P}^{1}$ and hence $|R-2 S|=\left\{S^{\prime}\right\}$ for some curve isomorphic to $S$. However, $(R-2 S)^{2}=4,(R-2 S) \cdot K_{X}=0$ imply that the arithmetical genus of $S^{\prime}$ is equal to 1 . This contradiction proves that the image of $S$ must be a fibre of a ruling on $\mathbb{P}^{1} \times \mathbb{P}^{1}$. This implies that $|R-2 S|$ is a pencil of elliptic curves (the pre-image of another ruling). Its image in $\mathbb{P}^{2}$ is a pencil of cubics with base points $q_{1}, \ldots, q_{9}$.

5.9. Theorem. Let $p_{1}, \ldots, p_{8}$ be general points in $\mathbb{P}^{5}$. Then the locus of point $p_{9} \in \mathbb{P}^{5}$ is such that $\operatorname{dim} E l\left(5 ; p_{1}, \ldots, p_{9}\right)=1$ is equal to the Weddle variety $W_{3}$ associated to the points $p_{1}, \ldots, p_{8}$.

Proof. We know that if $\operatorname{dim} \operatorname{El}\left(5 ; p_{1}, \ldots, p_{9}\right)=1$, then $\left(p_{1}, \ldots, p_{9}\right)$ is associated to a base point set of a pencil of cubics in $\mathbb{P}^{2}$. Now the assertion follows from 4.9 and the previous lemma.

5.10. Remark. The assertion of Theorem 5.9 agrees with (4.8) and Lemma 5.6. We have 4 Veronese surfaces $V_{i}$ on $E \in \operatorname{El}\left(5 ; p_{1}, \ldots, p_{8}\right)$. In each $V_{i}$ we find the ninth point $p_{9} \in E$ such that there exists a pencil of cubics through $p_{1}, \ldots, p_{9}$. The point $p_{9}$ must belong to $W_{3}$ and we have $E \cap W_{3}=4$.

5.11. Remark. It was shown by Coble [4, p. 489] that $W_{3}$ is of degree 19 with singular points at $p_{1}, \ldots, p_{8}$ of multiplicity 9 , triple lines $\left\langle p_{i}, p_{j}\right\rangle$ and the triple curve equal to the rational normal curve through the points $p_{1}, \ldots, p_{8}$. 
5.12. By Theorem 5.2 we have a rational elliptic fibration

$$
\Phi: \mathbb{P}^{5}-\rightarrow \operatorname{El}\left(5 ; p_{1}, \ldots, p_{8}\right)
$$

defined by the universal family of elliptic curves passing through the points $p_{1}, \ldots, p_{8}$. Its set of points of indeterminacy is the Weddle variety $W_{3}$. If we blow up $p_{1}, \ldots, p_{9}$ and then blow up the proper inverse transform $\tilde{W}_{3}$ of $W_{3}$ we expect to get a regular map $\tilde{\Phi}: \tilde{X} \rightarrow \operatorname{El}\left(5 ; p_{1}, \ldots, p_{8}\right)$. Let $\pi: \tilde{X} \rightarrow \mathbb{P}^{5}$ be the composition of these blow-ups. For each nonsingular point $p \in W_{3} \backslash\left\{p_{1}, \ldots, p_{8}\right\}$ the image of the line $\pi^{-1}(p)$ under $\tilde{\Phi}$ in $\operatorname{El}\left(5 ; p_{1}, \ldots, p_{8}\right)$ is the pencil of elliptic curves from $\operatorname{El}\left(5 ; p_{1}, \ldots, p_{8}\right)$ passing though $p$. Thus there are 4 rational curves through each general point in the variety $\operatorname{El}\left(5 ; p_{1}, \ldots, p_{8}\right)$. The restriction of $\tilde{\Phi}$ over each such curve is a rational elliptic surface corresponding to the associated set of points $\mathcal{Q}$ of $\mathcal{P}=\left(p_{1}, \ldots, p_{8}, p\right)$ in $\mathbb{P}^{2}$. It is isomorphic to the proper inverse transform $\tilde{V}$ under $\pi$ of a Veronese surface containing $\left(p_{1}, \ldots, p_{8}, p\right)$. In the Cremona action $\mathrm{cr}_{2,9}: W_{2,9} \rightarrow \operatorname{Bir}\left(P_{2}^{9}\right)$ the orbit of $\mathcal{Q}$ is fixed under the subgroup $G$ isomorphic to $H=\mathbb{Z}^{8} \rtimes(\mathbb{Z} / 2 \mathbb{Z}$ ) (see [7, p. 124]). Using (4.3), we obtain that the orbit of the points set $\mathcal{P}$ is fixed under the subgroup isomorphic to $H$ in the Cremona action $\mathrm{cr}_{5,9}$. In particular, we find that the group of pseudo-automorphisms of the blow-up $X(\mathcal{P})$ contains an infinite group $G^{\prime}$ isomorphic to $H$. Let $V^{\prime}$ be the proper inverse transform of the Veronese surface $V$ in $X(\mathcal{P})$. The projection $\tilde{X} \rightarrow X(\mathcal{P})$ defines an isomorphism $\tilde{V} \rightarrow V^{\prime}$. The group $G$ leaves $V^{\prime}$ invariant and its action on $V^{\prime}$ corresponds to the action of $G$ on $X(\mathcal{Q})$. Apparently (I have not checked it), the action of $H$ on each $\tilde{V}$ is the restriction of the automorphism group of the generic fibre of the elliptic fibration $\tilde{\Phi}$ isomorphic to $H$.

5.13. Remark. The fact that $X(\mathcal{P})$ has an infinite group of pseudo-automorphisms was used by S. Mukai [9] to construct an example of a linear action of the additive group $\mathbb{G}_{a}^{3}$ in the polynomial algebra $\mathbb{C}\left[T_{1}, \ldots, T_{9}\right]$ such that the algebra of invariants is not finitely generated.

\section{References}

1. Babbage, D.: Twelve associated points in [5]. J. Lond. Math. Soc. 23, 58-64 (1948)

2. Coble, A.: Associated sets of points. Trans. Am. Math. Soc. 24, 1-20 (1922)

3. Coble, A.: Algebraic Geometry and Theta Functions. Coll. Publ. v. 10, Am. Math. Soc. 1929 [New reprint: Amer. Math. Soc. Providence, 1968]

4. Coble, A.: A generalization of the Weddle surface, of its Cremona group, and its parametric expression in terms of hyperelliptic theta functions. Am. J. Math. 52, 439500 (1930)

5. Coble, A., Chanler, J.: The geometry of the Weddle manifold $W_{p}$. Am. J. Math. 57, 183-218 (1935)

6. Coble, A.: The geometry of the Weddle manifold $W_{p}$. Bull. Am. Math. Soc. 41, 209-222 (1935)

7. Dolgachev, I., Ortland, D. Point Sets in Projective Spaces and Theta Functions. Astérisque 165 (1988)

8. Eisenbud, D., Popescu, S.: The projective geometry of the Gale transform. J. Algebra 230, 127-173 (2000) 
9. Mukai, S.: Counterexamples to Hilbert's fourteenth problem for the 3-dimensional additive group. Publ. Math. RIMS 1343 (2001)

10. Semple, J., Roth, L.: Introduction to Algebraic Geometry. Oxford: Oxford Science Publ. 1987

11. Tyurin, A.: Special 0-cycles on polarized K3 surfaces. Izv. Akad. Nauk SSSR, Ser. Mat. 51, 131-151 (1987) 